\title{
Automated Toll Booth using Morphological Edge Detection Algorithm
}

\author{
Kausalya. K, Bhavadarini. S, Gayathri. A
}

\begin{abstract}
In order to reduce the traffic jam, save time and to diminish the money loss of 300 crores/year, an automated system is proposed; The intelligent traffic control management systems which explains the installation of automation in toll plazas, which is a step towards improving the monitoring of vehicles. The main purpose of this arrangement is to implement a system, which automatically identifies an approaching vehicle and records the vehicles number and time. If the vehicle belongs to an authorized person, the toll gate opens automatically and a predetermined amount is deducted from the user account, which leads to reduce the Traffic clogging at toll plazas. The vehicle number plate recognition is done only for authorized members. The proposed model consists of image digitization, edge detection, character detection and recognition with the payment transaction. The proposed method uses morphological edge detection, for the system to identify the character recognition and it uses template matching; it is done for license plates of 6 characters. The license plate character recognition system can be used in vehicle check -in and checkout monitoring system in hotels, malls and can be used to track vehicles on normal roads other than toll booths to avoid thefts.
\end{abstract}

Keyword: traffic jam, intelligent traffic control management, systems, vehicles, Traffic clogging

\section{INTRODUCTION}

As we all are aware the growth of a country depends upon its basic transportation. An express highway, for substantial movement, is one of them. But there are long queues at each toll plaza on the arterial highway, which wastes a lot of journey time, and fuel and emissions of co2.In our day to day life, we pay a certain amount of tax all the way through toll plazas. The toll gates are mostly found on national highways and bridges, etc., and we pay standing in a queue, and the mobility of vehicles gets affected. Therefore, in order to avoid this issue, we propose a system for automating the payments of toll by the using number plate recognition technique. Automating the toll booth increases its efficiency with the use of morphological edge detection and character segmentation algorithms, to detect and recognize license plates. Segmentation is done to determine the edges of the recognized number plate. Each character of the cropped number plate needs to be split and the acquired image is the resultant image.

Manuscript published on November 30, 2019

* Correspondence Author

Mrs. K.Kausalya*, Assistant Professor, Department of Information technology, Easwari Engineering college, Chennai, Tamilnadu.

Ms. Bhavadarini S, Studying, Easwari Engineering college, Chennai, Tamilnadu.

Ms. Gayathri, Studying, Easwari Engineering college, Chennai, Tamilnadu.

(C) The Authors. Published by Blue Eyes Intelligence Engineering and Sciences Publication (BEIESP). This is an open access article under the CC-BY-NC-ND license http://creativecommons.org/licenses/by-nc-nd/4.0/
It identifies separate objects within the image. Character Recognition is performed by the correlation matching algorithm implementation and the accuracy rate in the approximation of value 1 in the image [3]. Post recognition of vehicular ID, the details of the proprietor and the linked bank accounts are collected from the database. The toll fee is deducted from the bank account if the amount is available; else the fee is manually paid. If more than one vehicle belongs to a particular organization in nearby tolls then these vehicles are clustered to reduce the number of transactions made. One vehicle is chosen as a cluster head and this vehicle signifies all the cluster members and an ID based Multi signature is used for authentication [6]. The efficiency of the system is enhanced and reduces the time consumed.

The proposed system requires a high resolution camera to acquire an image of the vehicle. The proposed system consists of seven modules. They are Image Acquisition; Image pre-processing, Feature Extraction, Image Segmentation, License Plate Detection and Recognition, and Payment Transaction.

\section{EXISTING SYSTEM}

In the existing system, a toll collector enters the car details, receives the cash and acknowledge with a receipt with ease [10]. The drawbacks of the existing system are the processing time is high; the toll collector's efficiency determines the total efficiency of the system; manual cash payment takes more time and highly crowded tolls need more human effort [8].

\section{PROPOSED SYSTEM}

In the proposed system, image processing techniques such as morphological edge detection and character segmentation are used to identify the vehicles for an automated toll booth. Morphological edge detection methods are used to detect the license plate of the vehicle and Character Segmentation Algorithm (CSA) is used to recognize the license plate number. Theft vehicles can be detected and stopped with the reference of the police database. In case a theft vehicle is detected, a buzzer will be alarmed. Toll charges are deducted from the bank account synchronized with the vehicle. Toll charges are deducted based on the type of vehicle. The vehicle detail is obtained from the RTO database.

The proposed method has several plusses;

- Reduces the crossing setback.

- Faster and more efficient service

- Improved the convenience and console. 
- No need to wait for the change from the toll collector.

- No necessity to wind down the window.

- The toll authority receives payment in advance which enhances the toll auditing competence.

- The facility to make payments by keeping a balance in the bank account.

- It improves the public outlook to tolling.

The intended system consists of the following steps: image acquisition, gray-scale conversion, image preprocessing, character recognition,and payment transaction [2].The main advantage of the proposed system is that the Edge detection method produces better results than canny and Sobel methods. It is more reliable and quicker.

\section{A. Architecture Design}

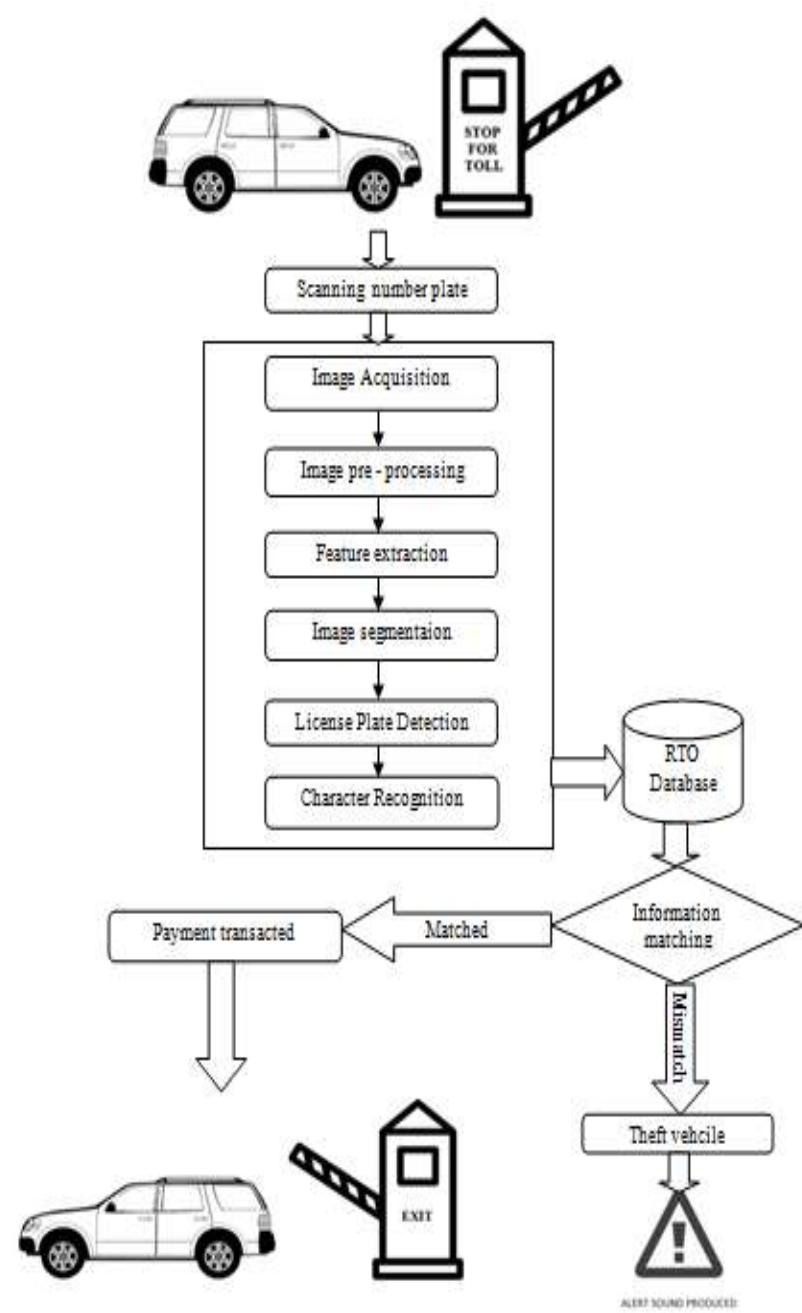

Fig No: 1 Architecture design of the proposed system

\section{B. Modules In the Proposed System}

In the Proposed System, for an automated toll booth, the whole description of the process is shown. The first part describes how the image of the car is captured based on the wheel arriving. In the first step of the workflow sequence no processing is possible without an image. Then the license plate's location is identified; if the license plate appears, then check the license number; if yes, the license plate is identified and the flow chart is shown below in Fig 2.

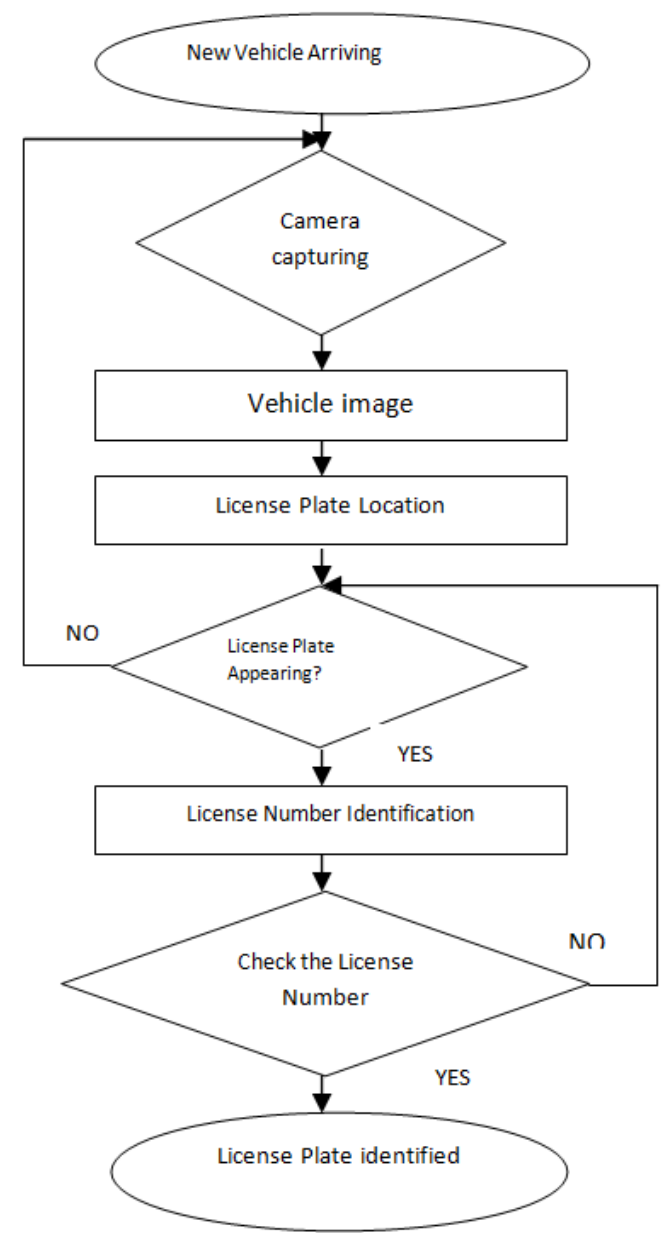

Fig No:2 Flow chart for the proposed system.

The vehicle enters the automated toll booth, then the license number is checked, then the number plate is read and checked with the police database. Then the balance is checked with the recognized number plate. If the required amount is available, then the vehicle is checked from the stolen vehicle the database; if the vehicle is stolen then proper action is taken. If not the toll charge is deducted from the balance and finally the gate is opened. The flow of the process is shown in the flowchart from Fig 3.

The image which is captured from the car undergoes with the following processes. In Image pre-processing, the noise of the image is removed using filters. Enhancement of image without noise is done, In Feature extraction, the dimension is reduced to the initial set of measures and builds the desired values intended to be informative and non-redundant. In Image Segmentation, the process of partitioning a digital image into multiple segments and simplifying them is done in a more meaningful manner. In License plate detection and recognition, the license plate is detected and recognized. The morphological edge detection methods are used to detect the edges over the image. In Character recognition, a method is used to detect the characters. Connected component labelling segments the license plate number. The detected license plate number is searched against the databases for toll payment. A payment transaction is present to deduct the charges for the particular vehicles which pass the toll booth. 
The database is used to maintain the records of the vehicles which pass through the toll. All the transactions of the particular vehicle are stored in the database.

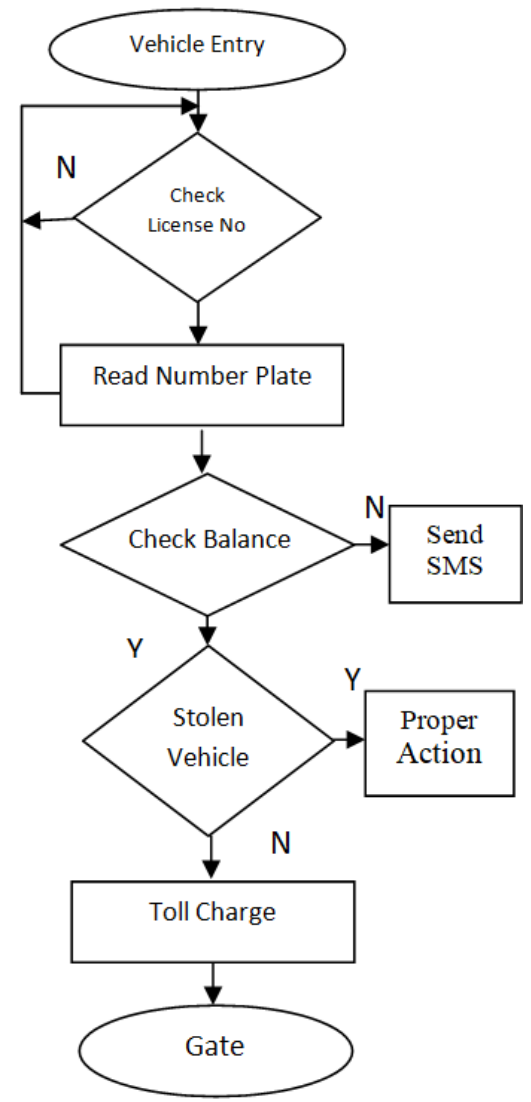

Fig No: 3 Flow chart for recognition of number plate

\section{The overall system is proposed}

\section{A Image Acquisition}

Image acquisition is the creation of the interior structure of an object; it includes processing, storing and displaying the image. In image processing ,Image acquisition can be broadly defined as the act of retrieving an image from some basis, usually a hardware-based source, so that it can be passed through whatever processes need to occur later[5]. Performing image acquisition from fig 4 in image processing is always the first step in the workflow sequence because, without an image, no processing is possible. The input image is given to the system for processing.

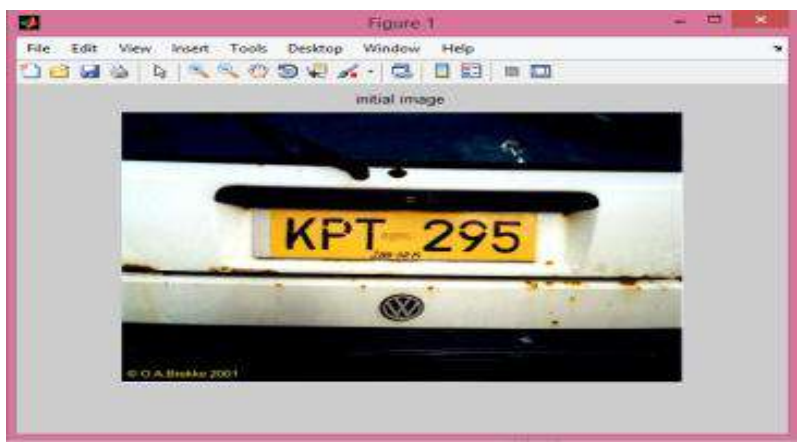

Fig No: 4 Image Acquisition

\section{B Image processing Unit}

Grayscale transformation: In Fig 5, the recognized input image is converted into a gray scale image. In 24-bit color image, each and every pixel contains the $\operatorname{Red}(\mathrm{R})$, Green $(\mathrm{G})$ and Blue (B) color components, each and every consuming 8 bits of information. From these R, G, B components, 8-bit gray value for each pixel position is calculated using the formula,

$$
\mathrm{a}=0.299 * \mathrm{R}+0.578 * \mathrm{G}+0.114 * \mathrm{~B}
$$

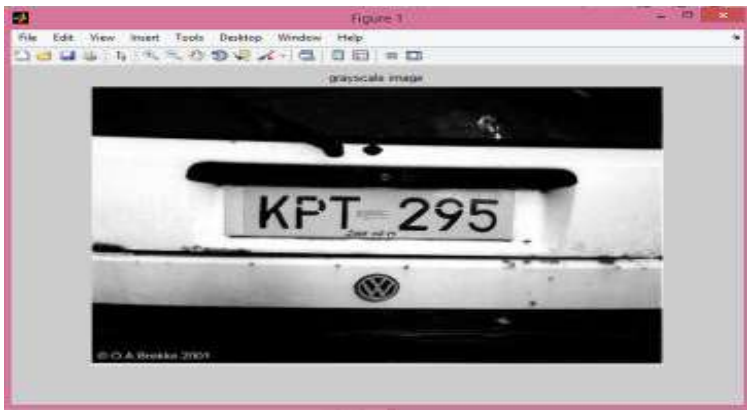

Fig No: 5 Gray scale conversion

C.Image Pre-processing:

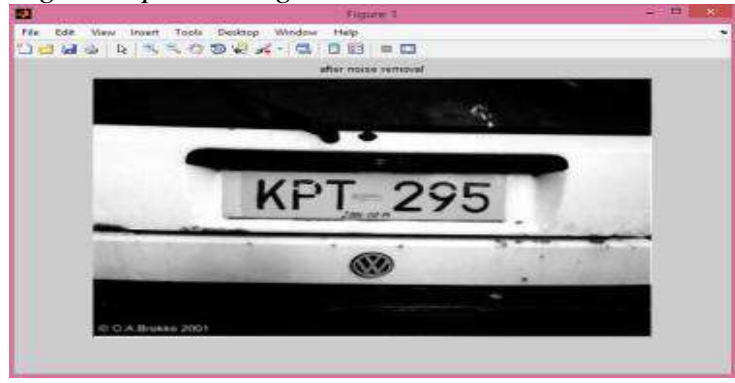

Fig No: 6 Noise removal

D.Feature Extraction: It involves reducing the quantity of resources needed to explain an outsized set of information.From the measured initial data set, Feature extraction starts and builds resulting values (eatures) projected to be enlightening and non-redundant, ease the consequent learning and generalization steps, and in some cases leading to better human understanding. Feature extraction is related to dimensionality reduction. In the proposed system, feature edge is extracted from the image acquired. Fig No: 7, shows the image after double conversion.

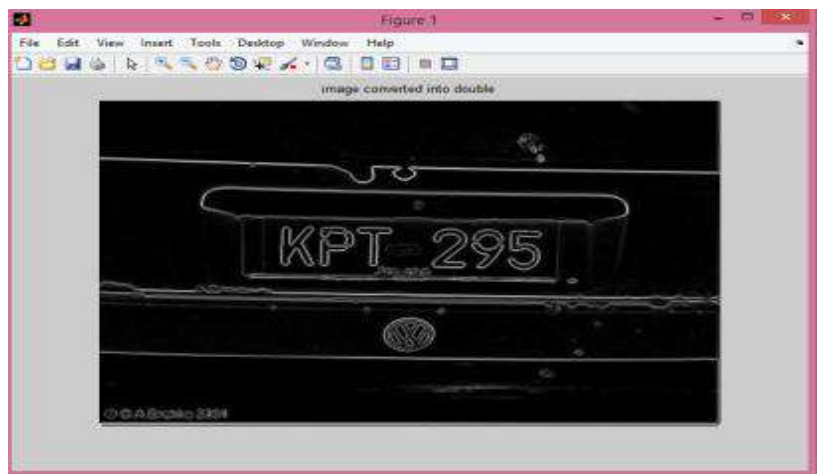

Fig No: 7 Double Converted Image

E.Image Segmentation: In Image segmentation, the digital image is partitioned and processed into multiple segments including sets of pixels also known as super pixels.

Published By: 
The objective of segmentation is to abridge and change the depiction of an image into a

clear representation that is easier to analyze. Segmentation is typically used to locate objects and boundaries (lines, curves, etc.) in a converted image. Image segmentation mainly focuses on detection of Discontinuities. There are various detection techniques, like Point detection, Line detection, Edge detection, combined detection, Edge linking and boundary detection [4].

F:License Plate Detection and Recognition: In this module, morphological edge detection and the character segmentation algorithm is used to detect and recognize the license plate [12]. The morphological edge detection methods such as erosion, dilation, open and close are used to detect the edges over the image. From that, the license plate of the vehicle is detected [11]. It removes all the small objects present in the image. Fig no: 8 shows the Image connecting edges and removing of small objects.

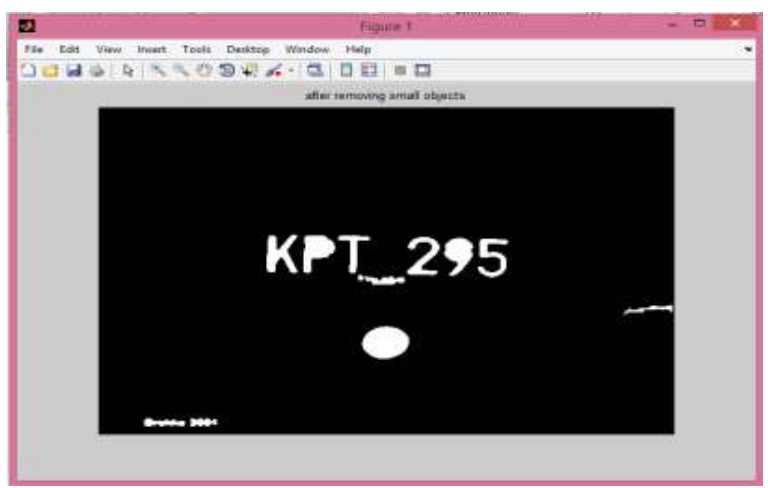

Fig No: 8 Image after removing small objects

G:Character Recognition:Character recognition enhances the processing of scanned images, it automatically recognizes and extracts the text content from the different data fields [9]. Connected Component Labeling (CCL) is used to detect and segment the license plate number. The outcome of the CCL algorithm is the set of foreground segment, which is the license plate number. The detected license plate number is searched against the databases for toll payment. Fig No:9 shows the license plate number in the notepad.

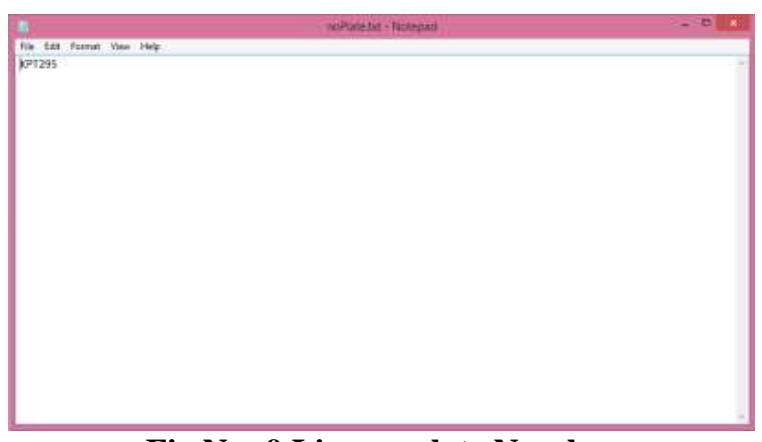

Fig No: 9 License plate Number

H:Payment Transaction:In this module, the toll charges are debited from the registered bank account. The toll charge is debited based on the type of vehicle. The vehicle type is obtained from the RTO database.
Identifying Theft Vehicles:In the proposed system, a supplementary feature of identifying theft vehicles has been added. The vehicle's license number is searched against the database. If the vehicle is found to be stolen, then an alert message and sound is produced. Fig No: 10 shows the warning message of a stolen vehicle.

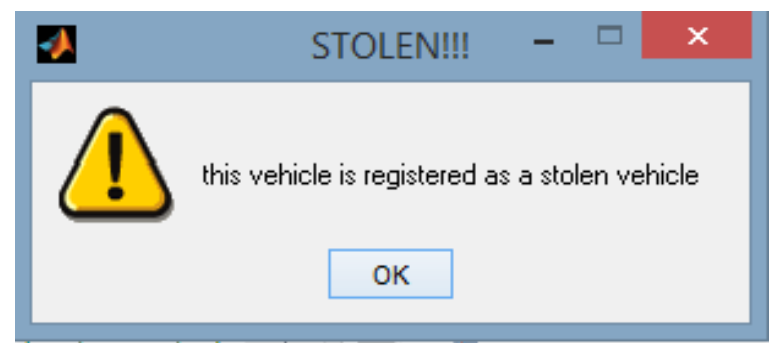

Fig No: 10 Warning Message for Stolen vehicle

I:Displaying the result:In this module, the vehicle details like license number, owner's name, vehicle type, toll, charge for the vehicle, and Account number are displayed over the GUI. Fig no: 11 demonstrates how the GUI displays the details of the vehicle.

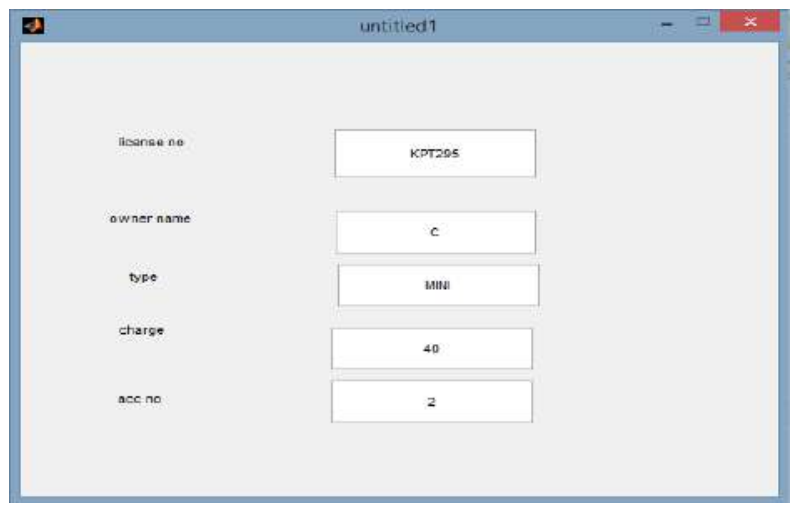

Fig No: 11 GUI to display the vehicle information

\section{EXPERIMENTAL RESULTS AND DISCUSSIONS}

In Fig No 12, the evaluation of the efficiency,of the system has been tested with images acquired through Logitech c270 web camera. The images have been acquired from the front and sides of the car and the system is able to recognize the license number. The images of the car are taken along with the lighting effects during night time.

The performance can be increased by using a better camera and lighting system. [1] The Paper presents a grouping of theoretical practice and the visual results on the vehicle number plate recognition through the morphological edge detection and proposed Otsu's algorithm for the segmentation process. The approximate results depict that it recognized the character with high accuracy, but there is no approach for the stolen vehicle and feasible system for reducing the human effort. M.Shehata,[7] introduces an approach for automatic identification of the vehicle's license plate. The character recognition is done by ANN and it is proposed that the ANN method was better than the Template matching method.

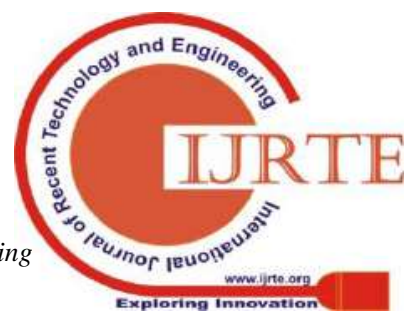


There are certain restrictions on the parameters which have low accuracy on the quality of the image and skew in the image. The proposed system has overcome the restrictions and produces high accuracy image for further processing.

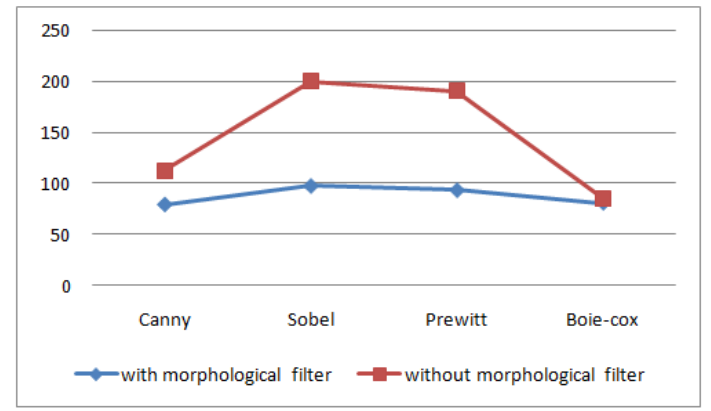

Fig No: 12 Comparisons between with and without morphological filter

\section{CONCLUSION AND FUTURE STUDY}

In this paper, we have presented a simple and efficient system for an automated toll booth based on a morphological edge detection algorithm. The system is able to recognize a license plate number with ease during the day and needs a bit more enhancement for recognition of the license plate number during the night, and the system is prepared for license plate characters with a maximum of 6 digits. A future study can be carried out over this system to recognize characters having up to 10 digits and can make enhancements to the image acquisition module during the night.

\section{REFERENCES}

1. Vehicle Number Plate Recognition Using Morphological Edge Detection and Segmentation-Volume 5, Issue 1" by Teena Singh Automatic Rajput in International Journal of Emerging Technology and Advanced Engineering on January 2015.

2. M. Ibrahim and M. Shehata, "Automatic license plate recognition (ALPR): A state-of-art review," IEEE Transaction on Circuits and Systems for Video Technology, Vol. 23, NO. 2, February 2013.

3. Improved Algorithm of Edge Detection Based on Mathematical Morphology Yao- Vol no-7, No.5 (2014) pig: 309-322 ” by Deng Caixia, Chen Yu, Bi Hui and Han in International Journal of Signal Processing and Pattern Recognition.

4. "Image Processing Based Automatic toll Booth in Indian conditions" by Priyanka Chhoriya, Govinda Paliwal, Poonam Badhan in International Journal of Emerging Technology and Advanced Engineering on April 2013.

5. Vehicle_Registration_Plates_Of_India.Available:http://en.wikipedia. org/wiki/Vehicle_registration_plates_of_india.

6. Shan Du, Member,IEEE, Mahmoud Ibrahim, Mohamed Shehata, Senior Member, IEEE and Wael Badawy, Senior Member, IEEE. Automatic License Plate Recognition. State of the art Review, Available at ieeexplore.org, Vol 23,07 June 2012.

7. Ankush Roy Debarshi, Patanjali Ghosal, Number Plate Recognition for Use in Different Countries Using an improved segmentation, Available at http;//ieeexplore.ieee.org,vol2,june 2012

8. S. Karthi, K. Pranathi, A. Srisaila, Automatic Number Plate Recognition, available at http;//ijict.org/, Vol2, No3 July 2011

9. Subhas Tatale.Akhil Khare,Real time ANPR for vehicle identification using neural network, ijaet,vol.1, Issue 4,pp.262268, sept 2011

10. M.Ibrahim and M.Shehata, Automatic license plate recognition a state of art review, IEEE Transaction on Circuits and System for Video Technology, Vol. 23,NO.2, February 2013.

11. Kumar Parasuraman, Member, IEEE and Subin P.S "SVM Based License Plate Recognition System" 2010 IEEE International Conference on Computational Intelligence and Computing Research

12. Ragini Bhat1, Bijender Mehandia2 "RECOGNITION OF VEHICLE NUMBER PLATE USING MATLAB" international journal of innovative research in electrical, electronics, instrumentation and control engineering.

13. K. Kausalya, S. Chitrakala" Idle Object Detection in Video for Banking ATM Applications" Research Journal of Applied Sciences, Engineering and Technology 4(24): 5350-5356, 2012.

\section{AUTHORS PROFILE}

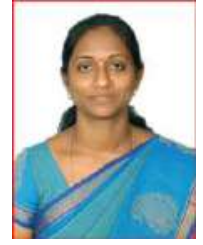

Mrs.K.Kausalya, completed her M.E in anna university,Chennai. Published 5 journal papers and 6 internal conferences papers.Now,Currently working as a Assistant Professor, Department of Information technology in Easwari Engineering college,Chennai,Tamilnadu. Her area of interest in research are Image processing,video Processing and data mining.

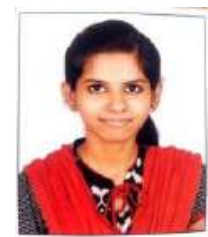

Ms.Bhavadarini S, is studying in Easwari Enginnering college.Her area of interest are Image processing and data mining.

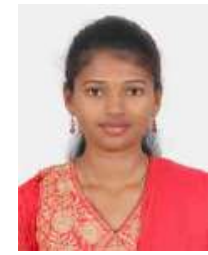

Ms.Gayathri, is studying in Easwari Enginnering college.Her area of interest are Image processing and data mining. 